\title{
Single-Cell Isolation and Size Sieving Using Microenclosure Array for Microbial Analysis
}

\author{
Akihiro Matsutani* and Ayako Takada ${ }^{1}$ \\ Semiconductor and MEMS Processing Center, Tokyo Institute of Technology, \\ 4259 Nagatsuta, Midori-ku, Yokohama 226-8503, Japan \\ 'Biomaterial Analysis Center, Tokyo Institute of Technology, \\ 4259 Nagatsuta, Midori-ku, Yokohama 226-8501, Japan
}

(Received December 25, 2014; accepted February 2, 2015)

Key words: single-cell isolation, size sieving, microenclosure, microbial analysis, plasma etching

We demonstrated a size sieving microbial cell separation apparatus consisting of multipillar microenclosures. The apparatus consists of two kinds of microenclosures that differ in size, namely, $4 \times 4 \mu \mathrm{m}^{2}$ enclosures with $0.2 \mu \mathrm{m}$ space between $1 \mu \mathrm{m}$ diameter pillars and $8 \times 8 \mu \mathrm{m}^{2}$ enclosures with $1 \mu \mathrm{m}$ space. Single cells of Escherichia coli and Saccharomyces cerevisiae were separately captured in smaller and larger microenclosures, respectively, just by dropping mixtures. Moreover, we examined whether incubation using the microenclosure structure was possible or not, and succeeded in the incubation of $S$. cerevisiae. Microlens arrays using prefabricated mesa structures by the Ar plasma etching process were also manufactured. We believe that the fluorescence analysis or investigation of photochemical reactions for microbial single cells can be carried out by a combination of the microenclosure structure and optical devices.

\section{Introduction}

Conventional biochemical techniques, i.e., bulk analyses, can provide only average values from large numbers of cells. A method of manipulating single cells is required because we have realized that each cell shows great heterogeneity even in the same culture. In other words, a simple and superior single-cell isolation technique with functions of supplying and circulating liquid is required for the single-cell analyses of small cells such as microorganisms. For this purpose, microwell array devices are widely used for cell isolation. ${ }^{(1-4)}$ However, microparticles tend to aggregate by selfassembly. The phenomenon is called the "coffee ring effect". Generally, it is difficult to control a droplet of evaporating water, because it is a nonequilibrium system. Yunker et $a l$. have reported on the suppression of the coffee ring effect by shape-dependent capillary interactions using ellipsoids of polystyrene particles. ${ }^{(5)} \mathrm{Hu}$ and Larson demonstrated the

*Corresponding author: e-mail: matsutani.a.aa@m.titech.ac.jp 
Maragoni effect in coffee ring effect depositions using poly(methyl metacrylate) (PMMA) particles. ${ }^{(6)}$ Nguyen et al. examined the evaporation kinetics and the particle deposits of silica nanoparticle suspension droplets on a smooth hydrophobic surface. ${ }^{(7)}$ On the other hand, the coffee ring effect using bacterial cells has been investigated. ${ }^{(8)}$ As mentioned above, it is difficult to isolate bacterial cells in droplets on flat surfaces. Thus, we demonstrated the single-cell isolation of bacteria using a microenclosure array consisting of many micropillars as shown in Fig. 1.(9,10) However, it is important to separate bacterial cells or microorganisms according to size for application to biochips such as microchannel or $\mu$-total analysis system (TAS) devices.

In this paper, we report on a novel biochip for size sieving and single-cell isolation using multipillar microenclosures.

\section{Single-Cell Isolation and Size Sieving of Cells}

\subsection{Principle of single-cell isolation and size sieving of cells}

Figure 2(a) shows a schematic of the proposed structure for the size sieving microbial cell isolation apparatus. First, we fabricated the optimized structure in pitch and space between micropillars to capture microorganisms of different sizes. Next, the separation of bacterial cells or microorganisms was achieved by dropping a liquid containing microbial cells of various sizes. In the proposed structure, for example, Saccharomyces cerevisiae cells can be captured in the larger microenclosures but not in the smaller ones. On the other hand, Escherichia coli cells can enter into both microenclosures but escape from the larger ones by liquid flow through micropillars. The liquid flow can be directed by tilting chips or sucking with a filter paper as shown in Fig. 2(b). The suction speed of a suspension of polystyrene beads by filter paper was about $1700 \mu \mathrm{m} / \mathrm{s}$.

\subsection{Examination of size sieving using polystyrene beads}

Figure 3 shows an optical microscopic image of sieving of polystyrene beads of different sizes. For fabricating the proposed structure, we used an electron beam lithography system (Elionix ELS6600) and an inductively coupled plasma (ICP) etching

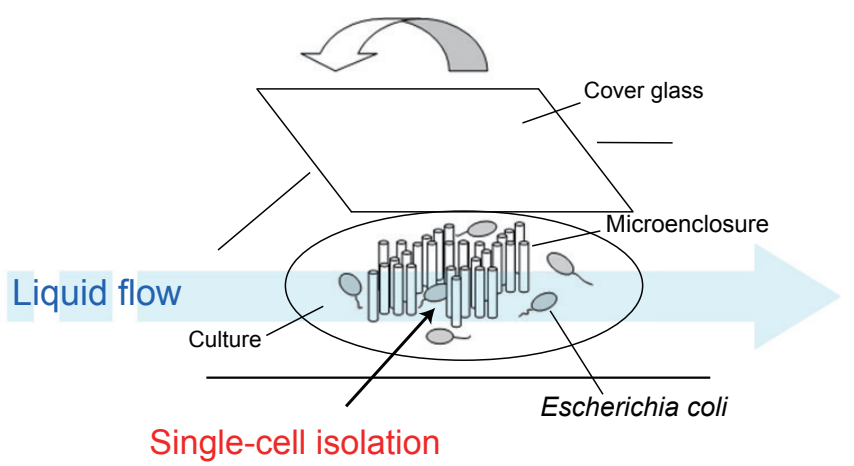

Fig. 1. (Color online) Concept of single-cell isolation of bacteria using a microenclosure. 




(a)

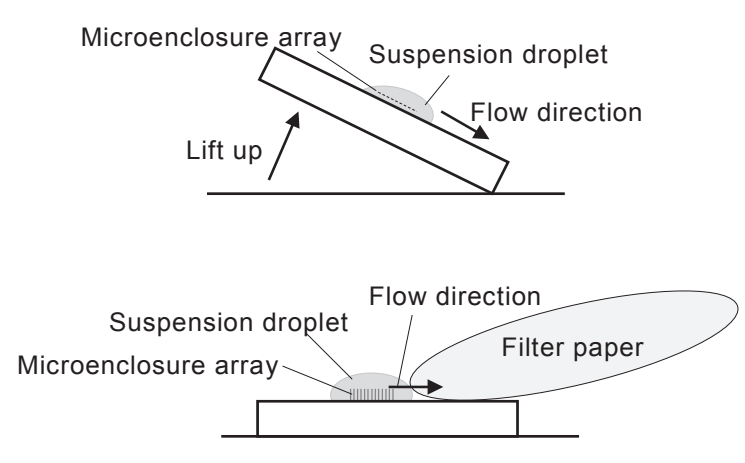

(b)

Fig. 2. (a) Schematic of proposed structure for size sieving microbial cell isolation apparatus and (b) liquid flow directed by tilting chips or sucking with a filter paper.

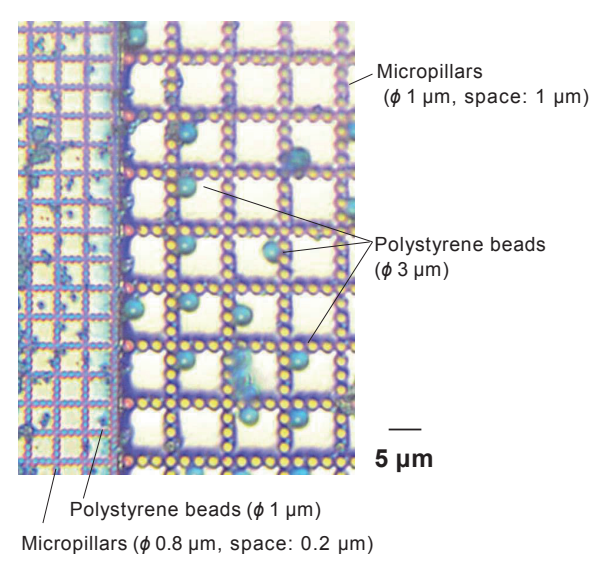

Fig. 3. Optical microscopic image of sieving of polystyrene beads.

system (Samco RIE-101ip) with $\mathrm{Cl}_{2}$ as an etching gas.(11-13) In addition, we used an InP wafer as a base material of the microenclosure array for combination with optical devices as discussed in the last chapter. We used polystyrene beads of 1 and $3 \mu \mathrm{m}$ diameters to confirm the efficiency of the proposed structure. The pitch and diameter of the micropillar array for the $1 \mu \mathrm{m}$ beads were 1 and $0.8 \mu \mathrm{m}$, and those for the $3 \mu \mathrm{m}$ beads were 2 and $1 \mu \mathrm{m}$, respectively. In other words, the space between micropillars for the $1 \mu \mathrm{m}$ beads was $0.2 \mu \mathrm{m}$, and that for the $3 \mu \mathrm{m}$ beads was $1 \mu \mathrm{m}$. In this structure, the $1 \mu \mathrm{m}$ beads cannot pass through the space of $0.2 \mu \mathrm{m}$, and the $3 \mu \mathrm{m}$ beads cannot pass through the space of $1 \mu \mathrm{m}$. However, the $1 \mu \mathrm{m}$ beads can pass through the space of $1 \mu \mathrm{m}$ of the microenclosure, and the $3 \mu \mathrm{m}$ beads cannot be trapped within the smaller microenclosure. Therefore, the $1 \mu \mathrm{m}$ beads and the $3 \mu \mathrm{m}$ beads were separately captured into smaller and larger microenclosures, respectively, as shown in Fig. 3. 


\subsection{Demonstration of size sieving for E. coli and S. cerevisiae cells}

Figure 4 shows an optical microscopic image of isolation of $E$. coli and $S$. cerevisiae cells. The pitch and diameter of the micropillar array for $E$. coli were 1 and $0.8 \mu \mathrm{m}$, and those for $S$. cerevisiae were 2 and $1 \mu \mathrm{m}$, respectively. The density of the bacterial cell suspension was $1 \times 10^{6} \mathrm{cells} / \mathrm{mL}$. The dropping volume was $1 \mu \mathrm{L}$. The typical size of $E$. coli cells is $1-2 \mu \mathrm{m}$, and that of $S$. cerevisiae is $3-5 \mu \mathrm{m}$. Therefore, E. coli cells cannot pass through the space of $0.2 \mu \mathrm{m}$, and $S$. cerevisiae cells cannot pass through the space of $1 \mu \mathrm{m}$. However, E. coli cells can pass through the $1 \mu \mathrm{m}$ microenclosure space for $S$. cerevisiae cells, and $S$. cerevisiae cells cannot be trapped into the smaller microenclosure. Thus, we can see that $E$. coli and $S$. cerevisiae cells were separately captured into smaller and larger microenclosures, respectively, as shown in Fig. 4.

\section{Incubation of Cells Using Microenclosure}

As mentioned above, we used an InP wafer, which is a III-V compound semiconductor for optoelectronic devices, as a material of the microenclosure. Although $\mathrm{SiO}_{2}$ or polyresin can be coated on the microenclosure chip by sputtering and evaporation techniques, it is important to confirm the chemical effect of the semiconductor material on bacterial cells. To determine this, we examined transformation efficiency on materials of the microenclosure by an electroporation method using E. coli. Transformation efficiencies on InP and GaAs substrates were comparable to that of the blank (if not insert the substrate material). Therefore, we believe that $\mathrm{InP}$ and GaAs substrates can be used as microenclosure materials for microbial analysis.

Prior to the incubation experiment, we confirmed whether the InP substrate is etched by yeast petone dextrose (YPD) media. It was found that the InP substrate was not etched by a culture solution of S. cerevisiae. The $\mathrm{pH}$ of the media was 5.7 after $6 \mathrm{~h}$ incubation. Figure 5(a) shows the incubation system used in this experiment. We used a long working distance objective lens for the direct observation of the incubation with an optical microscope. The incubation stage temperature was maintained at $30{ }^{\circ} \mathrm{C}$ during incubation. The microenclosure chip was placed at the center of a $35 \mathrm{~mm}$ culture dish. S. cerevisiae was grown in YPD media and cell culture $\left(4 \times 10^{6}\right.$ cells $\left./ \mathrm{mL}\right)$ of $2 \mathrm{~mL}$ was

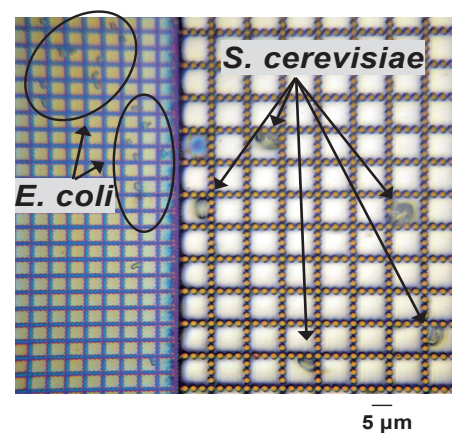

Fig. 4. Optical microscopic image of isolation of E. coli and S. cerevisiae cells. 


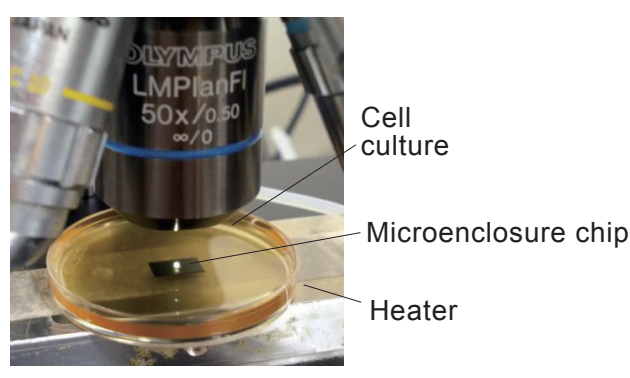

(a)
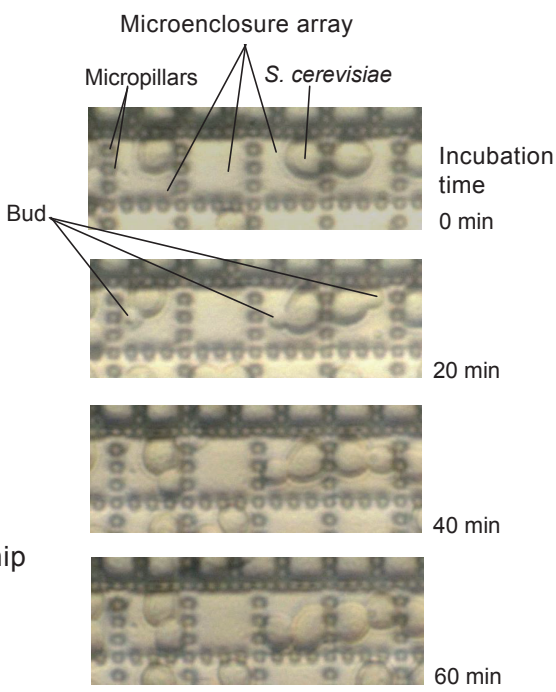

(b)

$$
\overline{5 \mu \mathrm{m}}
$$

Fig. 5. (a) Incubation system used in this experiment and (b) results of a $1 \mathrm{~h}$ incubation of $S$. cerevisiae with the microenclosure array.

poured into the dish. Figure 5(b) shows the results of a $1 \mathrm{~h}$ incubation of $S$. cerevisiae with the microenclosure array. It was demonstrated that the single isolated yeast cells can be incubated in the trapped state in the microenclosure. It was also observed that the isolated $S$. cerevisiae was cultured without protruding into neighboring microenclosures. This result suggests that the microenclosure array can be applied to the incubation of various kinds of microgranisms by optimizing the structure and size of micropillars. From the above results, we believe that the single-cell isolation chip, which gives hardly any damage to the cells, was realized using the micloenclosure structure.

\section{Prospect of Combination with Optical Devices and Single-Cell Isolation Structure}

We would like to propose an application with optical devices using the size sieving cell separation chip mentioned above. The vertical-cavity surface-emitting laser (VCSEL) is a semiconductor laser that can be monolithically fabricated. ${ }^{(14)}$ The VCSEL is now considered to be an important device for optical interconnects. We believe that the VCSEL can also be used as a device for sensoring or analysis. Thus, we propose a single-cell isolation technique on the VCSEL wafer as shown in Fig. 6(a). It can be used for single-cell fluorescence analysis or photochemical reaction. In addition, application to sensing devices and cell manipulation can be expected. Figure 6(b) shows a scanning electron microscopy (SEM) image of the single-cell isolation of $S$. cerevisiae cells captured on a VCSEL wafer by the dropping process. It was found that single $S$. 


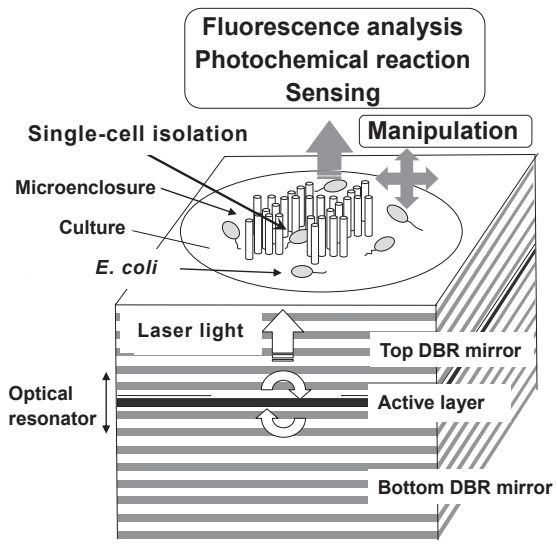

(a)

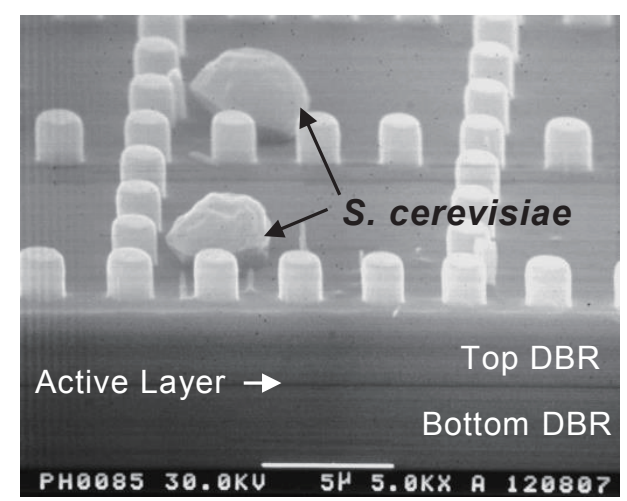

(b)

Fig. 6. (a) Schematic of single-cell isolation technique on the VCSEL and (b) SEM image of the single-cell isolation of $S$. cerevisiae cells captured on VCSEL wafer.

cerevisiae cells were captured in each microenclosure. Therefore, we believe that the hybrid systems, which combine the proposed structure and optoelectronic components, would become important devices for applications in neuroscience, medicine, and information technology.

The optical trapping technique using lasers is used for small particles. ${ }^{(15,16)}$ This technique is based on the force of radiation pressure. In this method, small particles or cells are trapped using a light beam focused with an objective lens of a microscope. On the other hand, parallel transport of biological cells can be performed using individually addressable VCSEL arrays as optical tweezers. ${ }^{(17)}$ In order to efficiently trap and transport cells, it would be convenient to use numerous spots focused by a microlens array. A microlens is generally fabricated by various techniques such as wet plasma processing, focused ion beam (FIB) technology, wet etching and laser lithography. ${ }^{(18-21)}$ However, the profile of a microlens fabricated by these techniques is affected by factors such as etching resistance and reflow shape of the resist. To improve the reproducibility of the microlens profile, we examined the microlens fabrication process using vertical mesa structures formed on a quartz substrate. The corners of the vertical profile mesa structure are etched preferentially by ions in the etching process. The reason is derived from the angular dependence of the sputtering efficiency.(22) We attempted microlens fabrication by utilizing this phenomenon. Figure 7(a) shows a schematic of the process flow in this experiment. A quartz substrate with a vertical mesa array pattern was used as a sample. We used a reactive ion etching system (modified Samco RIE-1). In addition, Ar plasma was used in this experiment. Figure 7(b) shows an SEM image of the quartz microlens array fabricated from a vertical mesa pattern structure. The diameter of the microlens was $0.5 \mu \mathrm{m}$. It is found that the microlens with a smooth surface profile was obtained by the proposed fabrication process. Figure 7(c) shows a photograph of the spot pattern focused by the fabricated microlens. A semiconductor laser diode of $670 \mathrm{~nm}$ wavelength was used as a light source. An array pattern of focused spots was observed. 


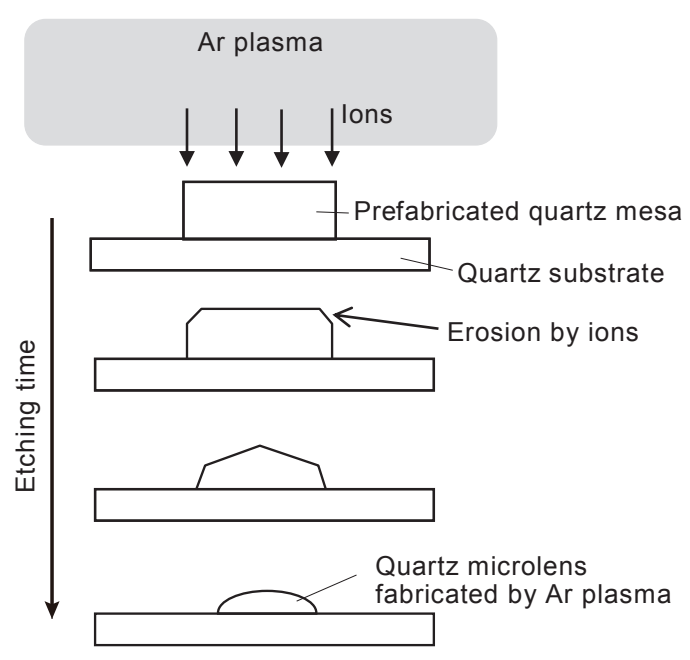

(a)

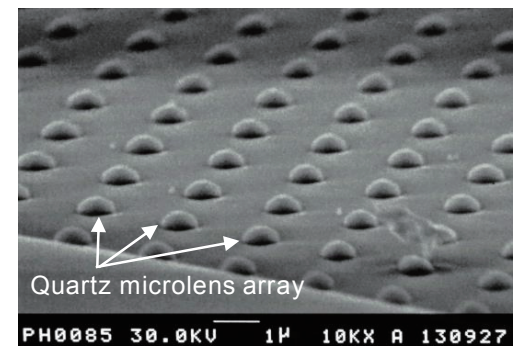

(b)

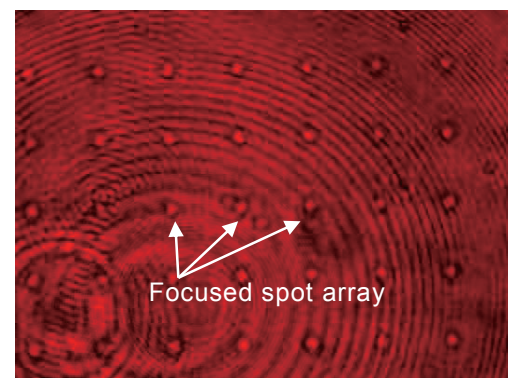

(c)

Fig. 7. (a) Process flow for fabrication of quartz microlens, (b) SEM image of quartz microlens array fabricated from a vertical mesa pattern structure, and (c) photograph of the spot pattern focused by the fabricated microlens.

The focal length was approximately $10 \mu \mathrm{m}$. Therefore, this fabrication technique is useful for fabricating a microlens array on optical device surfaces. We believe that the microenclosure structure can be widely applied for single-cell analysis by combining optical devices.

\section{Conclusions}

We proposed a novel biochip for single-cell size sieving of bacterial cells or microorganisms using a microenclosure with a multipillar structure. We noted that the polystyrene beads of various sizes were separately captured into smaller and larger microenclosures. In addition, we succeeded in ensuring that E. coli and $S$. cerevisiae cells were separately captured into smaller and larger microenclosures, respectively. Moreover, we succeeded in the single-cell isolation of S. cerevisiae into the microenclosure on the VCSEL wafer by a dropping process. For the various applications, we believe that the microenclosure chip can also be fabricated with $\mathrm{Si}$, glass, plastic, and poly(dimethylsiloxane) (PDMS) by using fabrication processes, such as nanoimprint lithography and conventional photolithography. We believe that the microenclosure structure can be widely applied for single-cell analysis by combining with optical devices. 


\section{Acknowledgements}

This work was supported by JSPS KAKENHI 26390037.

\section{References}

1 V. Chin, P. Taupin, S. Sanga, J. Scheel, F. Gage and S. Bhatia: Biotechnol. Bioeng. 88 (2004) 399.

2 S. Lindstrom, K. Mori, T. Ohashi and H. Anderson-Svahn: Electrophoresis 30 (2009) 1.

3 R. Iino, L. Lam, K. Tabata, Y. Rondelez and H. Noji: Jpn. J. Appl. Phys. 48 (2009) 08JA04.

4 I. Inoue, Y. Wakamoto, H. Moriguchi, K. Okano and K. Yasuda: Lab Chip 1 (2001) 50.

5 P. J. Yunker, T. Still, M. A. Lohr and A. G. Yodh: Nature 476 (2011) 308.

6 H. Hu and R. G. Larson: J. Phys. Chem. B 110 (2006) 7090.

7 T. A. H. Nguyen, M. A. Hampton and A. V. Nguyen: J. Phys. Chem. C 117 (2013) 4707.

8 W. Sempels, R. D. Dief, H. Mizuno, J. Hofkens and J. Vermant: Nat. Commun., DOI: 10.1038/ncomms2746 (2013).

9 A. Matsutani and A. Takada: Jpn. J. Appl. Phys. 49 (2010) 127201.

10 A. Matsutani and A. Takada: Jpn. J. Appl. Phys. 51 (2012) 087001.

11 A. Matsutani, H. Ohtsuki, F. Koyama and K. Iga: Jpn. J. Appl. Phys. 39 (2000) 1435.

12 A. Matsutani, H. Ohtsuki and F. Koyama: Jpn. J. Appl. Phys. 38 (1999) 4260.

13 A. Matsutani, H. Ohtsuki and F. Koyama: Jpn. J. Appl. Phys. 44 (2005) L576.

14 K. Iga: Jpn. J. Appl. Phys. 47 (2008) 1.

15 A. Ashkin: Proc. Natl. Acad. Sci. USA. 94 (1997) 4853.

16 H. Zhang and K. Liu: J. R. Soc. Interface 5 (2008) 671.

17 R. A. Flynn, A. L. Birkbeck, M. Gross, M. Ozkan, B. Shao, M. M. Wang and S. C. Esener: Sens. Actuators, B 87 (2002) 239.

18 A. Kouchiyama, I. Ichimura, K. Kishima, T. Nakao, K. Yamamoto, G. Hashimoto, A. Iida and K. Osato: Jpn. J. Appl. Phys. 40 (2001) 1792.

19 H. Tsuboi, M. Ito, M. Tanabe, T. Hayashi and T. Uchida: Jpn. J. Appl. Phys. 34 (1995) 2476.

20 Y. Fu, N. Kok and A. Bryan: Microelectron. Eng. 54 (2000) 211.

21 H. Nishiyama, J. Nishii, M. Mizoshiri and Y. Hirata: Appl. Surf. Sci. 255 (2009) 9750.

22 M. Cantagrel: J. Vac. Sci. Technol. 12 (1975) 1340. 